\title{
Embodiment of Sadness and Depression-Gait Patterns Associated With Dysphoric Mood
}

Johannes Michalak, PhD, Nikolaus F. Troje, Dr rer nat, Julia Fischer, Dipl-Psych, Patrick Vollmar, Dipl-Psych, Thomas Heidenreich, PhD, and Dietmar Schulte, PhD

\begin{abstract}
Objective: To analyze gait patterns associated with sadness and depression. Embodiment theories suggest a reciprocal relationship between bodily expression and the way in which emotions are processed. Methods: In Study 1, the gait patterns of 14 inpatients suffering from major depression were compared with those of matched never-depressed participants. In Study 2, we employed musical mood induction to induce sad and positive mood in a sample of 23 undergraduates. A Fourier-based description of walking data served as the basis for the computation of linear classifiers and for the analysis of gait parameters. Results: Gait patterns associated with sadness and depression are characterized by reduced walking speed, arm swing, and vertical head movements. Moreover, depressed and sad walkers displayed larger lateral swaying movements of the upper body and a more slumped posture. Conclusion: The results of the present study indicate that a specific gait pattern characterizes individuals in dysphoric mood. Key words: embodiment, gait, sadness, emotions, major depression, psychopathology.
\end{abstract}

ANCOVA $=$ analysis of covariance; BDI $=$ Beck Depression Inventory; DSM = Diagnostic and Statistical Manual of Mental Disorders; MANOVA = multivariate analysis of variance; MDD = major depressive disorder; SCID = Structured Clinical Interview for DSM-IV; SNRI = serotonin noradrenalin reuptake inhibitors; SSRI $=$ selective serotonin reuptake inhibitors.

\section{INTRODUCTION}

"He comes in with small tired steps, slowly takes a seat and sits there in a rather slumped posture, almost motionless, staring into space."

-E. Kraepelin, 1905, translated by J.M. (1).

$\mathrm{T}$ he way people move conveys a considerable amount of information. The expression of affective states via biological motion is a fundamental component of social interaction. People infer the mood states of other persons from dynamic movement patterns (2).

In addition to the fact that psychomotor information is relevant in social interactions between individuals, recent theories of emotion ${ }^{1}$ propose a reciprocal flow of information between bodily states (including that of the psychomotoric system) and emotional processes. These theories of emotion converge in the idea of the embodied nature of emotion $(3,4)$. The term "embodiment" is used by researchers in psychology (5), philosophy (6), artificial intelligence (7), and linguistics (8) to express the notion that knowledge is "embodied" or grounded

\footnotetext{
${ }^{1}$ The notion that bodily expression and emotional processes are closely interconnected can be traced back to the early days of academic psychology (35). Early critics of embodied emotion argued that bodily feedback is too undifferentiated and too slow to represent emotional experience (36). However, since then, research has shown that in fact the motor system can support extremely subtle distinctions (3).

From the Department of Clinical Psychology and Psychotherapy (J.M., J.F., D.S.), Ruhr-University Bochum, Germany; Queen's University (N.F.T.), Kingston, Ontario, Canada; (P.V.) Klinikum rechts der Isar, Technical University Munich; and University of Applied Sciences (U.H.), Esslingen, Germany.

Address correspondence and reprint requests to Johannes Michalak, Department of Clinical Psychology and Psychotherapy, Ruhr-University Bochum, 44789 Bochum, Germany. E-mail: Johannes.Michalak@rub.de

Received for publication August 18, 2008; revision received December 17, 2008.

Supported by a grant from the research fund of the center for psychotherapy of the Ruhr-University, Bochum, Germany, and a fellowship from the Human Frontiers Science Program (J.M.), as well as a Discovery Grant and a Steacie Fellowship from the Natural Sciences and Engineering Research Council of Canada (N.F.T.)
}

DOI: 10.1097/PSY.0b013e3181a2515c in bodily states and in the brain's modality-specific systems. Correspondingly, embodiment theories of emotion assume a complex reciprocal relationship between the bodily expression of emotion and the way in which emotional information is processed. According to these theories, experiencing emotional states affects somatovisceral and motoric systems whereas, on the other hand, bodily states have effects on how emotional information is processed. One example of a theory that stresses the importance of bodily information in depression is the interacting cognitive subsystems approach (9), which proposes a so-called "depressive interlock configuration." It assumes that bodily and cognitive feedback loops can become established that "lock" subsystems into a self-perpetuating configuration that maintains depression.

In our present research, we tried to elucidate one aspect of the complex interrelationship between body states and emotional processes. We focused on the effects that sad mood and depression have on the way people walk. We expected that there should be specific gait patterns associated with sadness and depression. Empirical research on biological motion has primarily focused on emotional behavior conveyed by facial expression (10). However, although the facial expression of emotions is important, it represents only a single aspect of emotion-related biological motion. There have only been few studies that investigate fullbody movements associated with depression and sadness. Some research has analyzed gait patterns in depressed patients (11-14). In sum, the results of these studies show that currently depressed patients evidence reduced gait velocity, stride length, increased standing phase and gait cycle duration (15). However, these studies have certain methodological limitations: they restrict their analysis to the lower limbs and to forward and backward limb movements, which means to one dimension.

Two studies investigated gait patterns associated with nonclinical forms of sadness and happiness. In a study by Montepare et al. (2) which primarily focused on identification of emotions from gait patterns, five walkers read brief scenarios describing emotional situations and were instructed to imagine themselves in the situations and to walk accordingly. Untrained participants rated gait parameters of the video displays of these walkers on dichotomous ad hoc scales (e.g., light-footed/ heavy-footed). Results indicated that participants imagining sad scenarios showed reduced arm swings and a marginally reduced stride length. However, it should be noted that the 
study of Montepare et al. (2) had several important methodological limitations (e.g., scales used, untrained raters, head was not displayed in the video tapes) so that the validity of the ratings may be seriously restricted. In a recent study, Omlor and Giese (16) tried to identify spatio-temporal patterns that are specific for different emotions. They asked lay actors to walk with four emotional styles (sad, happy, angry, fearful) and compared these styles with neutral walking patterns. They identified increases in elbow amplitude for happiness and decreases in elbow and shoulder amplitude for sadness. However, although this study utilized a sophisticated technology to analyze gait patterns (i.e., a nonlinear source separation technique), using the walking patterns of lay actors instead of actual emotions restricts the ecological validity of their results.

The aim of the present study was to analyze gait patterns in depressed and sad participants using complex movement analysis. In contrast to previous studies analyzing the gait patterns of depressed individuals, the present study employed a sophisticated technology that made it possible to conduct a comprehensive three-dimensional analysis of gait patterns associated with sadness and depression. Forward/backward movements, vertical movements, and lateral movements of all body segments were analyzed. In our first study, gait patterns of patients suffering from a current episode of major depressive disorder (MDD) were compared with a matched sample of never-depressed participants. In our second study, utilizing a within-subject design, we employed a musical mood induction procedure to induce sad and happy moods in a sample of undergraduate students. By comparing the results of the two studies, we wanted to estimate whether a convergent gait pattern characterizes sadness in nonclinical and major depression in a clinical population. By doing so, we tried to elucidate one aspect of the embodied nature of sadness and depression.

\section{STUDY 1}

\section{METHODS \\ Participants}

Participants were 14 psychiatric inpatients suffering from a current principal diagnosis of major depression and 14 never-depressed control participants matched for age and sex. The inpatients were recruited from two adult psychiatric units at university-affiliated hospitals. The units are specialized in the treatment of depression. The control participants were recruited through announcements and public notices. The mean ages were 44.2 years (range $=$ $29-61$ years) and 44.14 years (range $=30-64$ years) for the depressed participants and comparison group, respectively. In each group, six participants were male and eight female. They all voluntarily participated in the study.

To verify the diagnosis of the psychiatrists of the inpatient units, two trained raters conducted the German version of the Structured Clinical Interview for DSM-IV (SCID) (17). Patients were included in our study if they met the criteria of the Diagnostic and Statistical Manual of Mental Disorder (DSM-IV-TR, 4th TR edition) (18) for a primary diagnosis of current MDD. Additionally, we administered the Beck Depression Inventory (BDI) $(19,20)$. The BDI is a widely used 21-item self-report measure of the severity of depressive symptoms with good psychometric properties $(20,21)$. Only patients who scored $>12$ on the BDI were included in the depressed group.

Nine of 14 depressed patients had comorbid diagnoses (most of them had anxiety disorders). In the SCID, six patients reported symptoms of psychomotor agitation and four patients reported symptoms of psychomotor retardation.

Thirteen patients were receiving antidepressant medication. Six patients were being treated with selective serotonin reuptake inhibitors (SSRI), three patients with serotonin noradrenalin reuptake inhibitors (SNRI) whereas four patients were being treated with tricyclic antidepressants. Three patients were being treated with two antidepressants simultaneously (SSRI + tricyclic, SSRI + SNRI, SNRI + SNRI), one patient was receiving treatment with lithium as an augmental, one patient was receiving additional treatment with a neuroleptic agent, and four patients were receiving a nonbenzodiazepine hypnotic with a plasma half-time of 4 to 6 hours.

We also conducted the SCID with the participants from the comparison group. Only participants who had no current or lifetime affective disorder were included in the comparison group. All participants of the comparison group had BDI scores of $\leq 6$.

\section{Motion Data Acquisition and Procedure}

Motion data were collected with the motion capture system (Vicon 512, Oxford Metrics, UK) equipped with nine video cameras (Pulnix TM 6710, Oxford Metrics, UK) that made it possible to track small reflective markers in three-dimensional space with a spatial accuracy in the range of $1 \mathrm{~mm}$ and a temporal resolution of $120 \mathrm{~Hz}(22)$. A set of 41 markers were attached to the participants' bodies. The motion capture system was set up in a $90 \mathrm{~m}^{2}$ room. On arrival, participants were informed about the procedure and their written informed consent was obtained. They were also informed that they could terminate the experiment at any time.

Participants walked on a $7 \times 0.70$ meter red panel, which was placed within the elliptic capture volume. The following instruction was given to the participants: "This experiment investigates gait patterns. Please walk on this red rug. You can choose your walking speed freely." After they had walked back and forth at least 10 times, we started data recording.

The data of Study 1 were gathered between July 2004 and August 2005. The motion capture system permits a comprehensive three-dimensional analysis of motion data. Forward-backward movements (x-axis), lateral movements (y-axis), and up-and-down movements (z-axis) of the body can be recorded. We used the framework developed by Troje $(22,23)$ to transform the raw motion data into low dimensional representations to which methods from linear statistics can be applied. Fourier decomposition makes it possible to achieve efficient, low-dimensional, linear decompositions of the periodic motion data. It is nearly optimal in terms of covering a maximum of variance with a minimum of components.

Most of the earlier studies that analyzed gait patterns in persons with depression investigated particular gait properties suspected to be promising candidates for discriminating depressed from never-depressed participants. In our research, we chose a different approach (23) to the question as to which gait patterns are associated with depression. With no a priori assumptions about possible candidate gait properties, we computed a regression of a class identifier indicating whether a walker is depressive or never depressed (or, in Study 2, sad or happy) on the parameters of the walker's projection onto a linear morphable model. The resulting linear discriminant function was then scrutinized visually to determine which properties were used.

\section{RESULTS}

For Study 1 and Study 2, unless otherwise stated, all comparisons were based on dependent sample $t$ tests. A full description of the results is followed by a statement as to which analyses remained significant once a Bonferroni adjustment had been conducted to control for multiple $t$ tests.

\section{Participant Characteristics}

Depressed patients and never-depressed participants showed pronounced differences in the level of depressive symptoms (BDI; depressed: $\mathrm{M}=24.15, \mathrm{SD}=7.69$; never-depressed participants: $\mathrm{M}=2.07, \mathrm{SD}=1.90, t(13)=-9.47, p<$ .0001). Moreover, inspection of the participants' weight (in $\mathrm{kg}$ ) showed that depressed patients were heavier than neverdepressed participants (depressed: $\mathrm{M}=76.99, \mathrm{SD}=16.99$; 

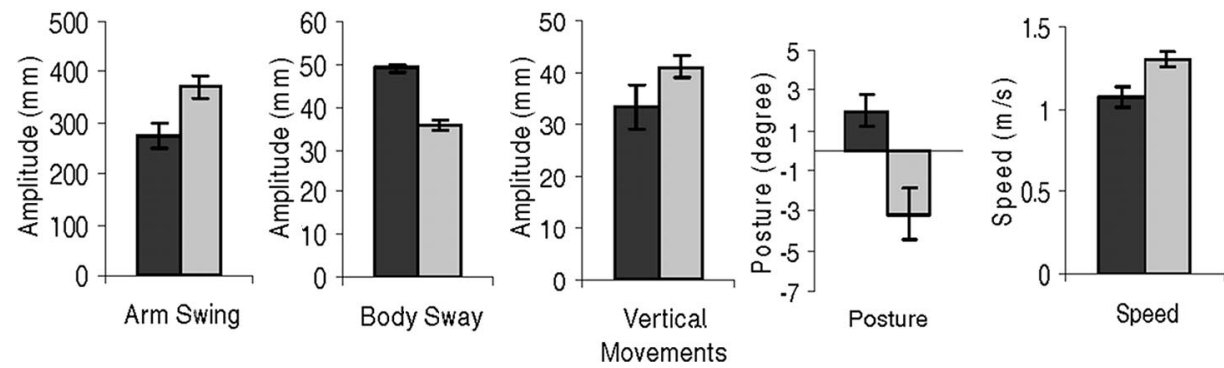

Figure 1. Study 1: Gait parameters of currently depressed and never-depressed participants. Error bars represent 1 standard error.

never-depressed participants: $\mathrm{M}=65.92, \mathrm{SD}=14.63, t$ $(13)=-4.11, p<.01)$. We refrained from attempts to statistically control weight as a covariate. Whereas analysis of covariance (ANCOVA) can be legitimately used as a technique to reduce noise (error) when groups do not differ in the covariate, attempts to statistically "control" substantial group differences are an invalid use of ANCOVA (24). When groups differ on a potential covariate, ANCOVA removes too much variance from the independent variable so that the grouping variable is altered in some substantive way not specifiable in a meaningful conceptual way. Thus, using weight as a covariate would have prevented meaningful interpretation of the group differences in gait characteristics. Instead, we correlated weight and gait characteristics in the group of depressed patients as an auxiliary analysis to assess the degree to which weight might contribute to the gait patterns.

\section{Gait Patterns}

A linear discriminant function was constructed by regressing group affiliation (14 depressed patients versus 14 neverdepressed controls) onto the parameters of the walker model (23). An animation visualizing this function can be viewed at http://biomotionlab.ca/Demos/BMLdepression.html. By visual inspection of the animation, we identified five features that most strongly differentiated the gait patterns of depressed and never-depressed participants. The decision was made by consensus between the first and the second author. The five features were speed (in $\mathrm{m} / \mathrm{s}$ ), arm swing (i.e., difference between extreme horizontal positions of the wrist markers in $\mathrm{mm}$ averaged across right and left hand side), lateral body sway (i.e., difference between maximum right and left deflection of the shoulder in $\mathrm{mm}$ ), posture of the upper body (i.e., angle between the line connecting clavicle and head marker and the vertical axis; a positive angle indicates that the head is in front of the clavicle marker), and vertical up-and-down movements of the upper body (measured in terms of the vertical amplitude of the head marker in $\mathrm{mm}$ ).

Figure 1 presents the mean and standard error values of the variables. There were significant differences between depressed and never-depressed participants for all five features: depressed patients walked more slowly (depressed: $\mathrm{M}=1.07$, $\mathrm{SD}=0.22$; never-depressed participants: $\mathrm{M}=1.30, \mathrm{SD}=$
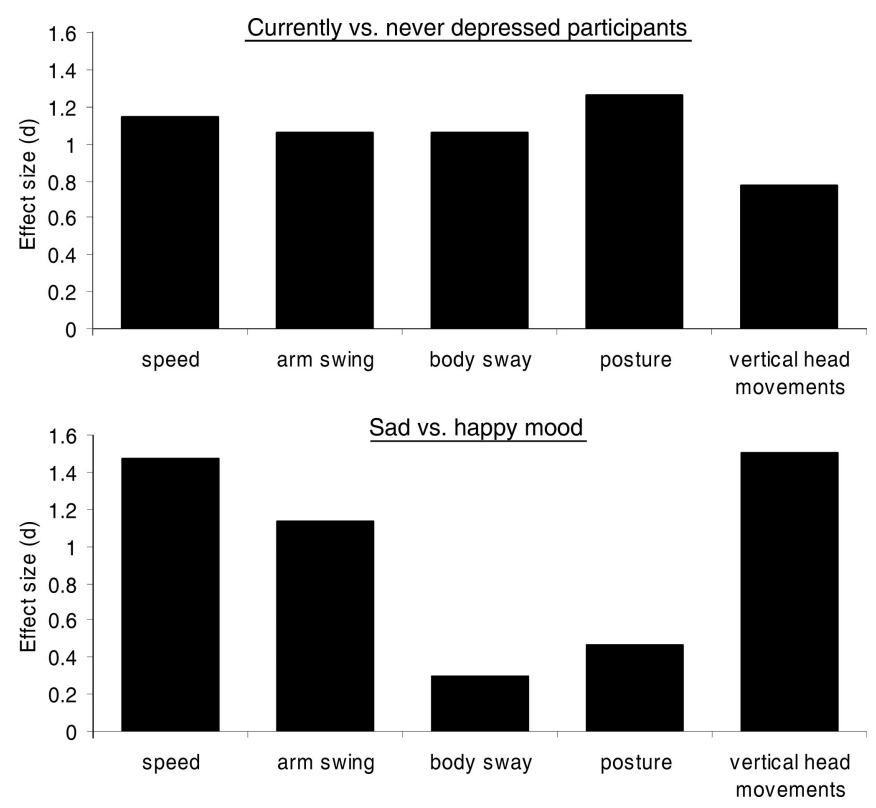

Figure 2. Effect sizes (d) for differences in gait parameters between currently depressed and never-depressed participants (Study 1, upper part) and between happy and sad mood (Study 2, lower part).

$0.17, t(13)=3.16, p<.01)$, showed reduced arm swing (depressed: $\mathrm{M}=274.07, \mathrm{SD}=97.62$; never-depressed participants: $\mathrm{M}=370.51, \mathrm{SD}=82.26, t(13)=2.693, p<.05)$, showed greater lateral body sway (depressed: $\mathrm{M}=49.03$, $\mathrm{SD}=16.11$; never-depressed participants: $\mathrm{M}=35.63, \mathrm{SD}=$ $7.91, t(13)=-3.59, p<.01)$, and exhibited a more slumped posture (depressed: $\mathrm{M}=1.97, \mathrm{SD}=2.99$; never-depressed participants: $\mathrm{M}=-3.17, \mathrm{SD}=4.93, t(13)=-3.57, p<$ $.01)$ and reduced vertical movements of the head (depressed: $\mathrm{M}=33.13, \mathrm{SD}=13.00$; never-depressed participants: $\mathrm{M}=$ $41.10, \mathrm{SD}=6.65, t(13)=2.26, p<.05)$. Following Bonferroni adjustment $(p<.01=.05 / 5)$, significance was maintained for speed, posture, and body sway.

As shown in the upper part of Figure 2, effect sizes (d) for the difference between depressed and never-depressed participants are large ( $d>0.80$ for speed, arm swing, body sway, and posture).

We conducted an additional exploratory analysis to assess the differences between the six patients predominantly show- 
TABLE 1. Mean (M) and Standard Deviation (SD) Values for Patients Predominantly Showing Psychomotor Agitation and Patients Showing Psychomotor Retardation

\begin{tabular}{|c|c|c|c|c|}
\hline \multirow{2}{*}{ Gait Characteristics } & \multicolumn{2}{|c|}{$\begin{array}{l}\text { Agitation } \\
(n=6)\end{array}$} & \multicolumn{2}{|c|}{$\begin{array}{l}\text { Retardation } \\
(n=4)\end{array}$} \\
\hline & M & SD & M & SD \\
\hline Speed & 1.2 & 0.28 & 0.96 & 0.13 \\
\hline Arm swing & 279.65 & 137.79 & 253 & 77.81 \\
\hline Body sway & 46.16 & 6.57 & 63.91 & 23.28 \\
\hline Posture & 2.12 & 2.66 & 3.09 & 4.05 \\
\hline Vertical movements & 38.27 & 18.93 & 30.47 & 5.54 \\
\hline
\end{tabular}

ing psychomotor agitation and the four patients showing psychomotor retardation. Because the small sample size precludes any extended analyses, we only report descriptive findings for these two subgroups in Table 1.

Because controlling for the effects of weight using ANCOVA is not feasible, we correlated weight and gait characteristics. The correlations between weight and the five gait characteristics were as follows: speed, $r=.29$ (NS); arm swing, $r=.29$ (NS); body sway, $r=.60(p<.05)$; posture, $r=.41$ (NS); vertical movements, $r=.54(p<.05)$. Heavier patients showed more pronounced lateral body sway and correspondingly a more "depressed" walking style. However, correlations between vertical movements and weight were the other way round. Heavier patients show more pronounced up-anddown movements, which are a characteristic of a "healthier" gait pattern. Although the correlation between the remaining gait characteristics and weight were nonsignificant, some of the directions of the correlations (i.e., positive correlations with speed and arm swing) remain inconsistent with the hypotheses that weight alone contributes to the differences between depressed and never-depressed individuals that we found in our present research.

\section{DISCUSSION}

The results of Study 1 revealed marked differences in gait patterns of currently depressed and never-depressed participants. Currently depressed patients walk more slowly than never-depressed control participants. This result is in line with previous studies showing reduced velocity and increased gait cycle duration in depressed patients (11).

In contrast to previous gait studies, which restricted their analyses to lower limb movements and one dimension, our study permitted a more comprehensive analysis of gait parameters. Although they are an element of case descriptions in the psychiatric literature (1), up to now characteristics such as slumped posture during walking, increased swaying movements in the upper body, and reduced vertical movements of the head have never - to our knowledge - been analyzed in research on gait patterns in patients with depression. The reduced vertical movements of the head are in line with the observation by Sloman et al. (13) that depressed patients walk with a lifting motion of the leg, whereas normal control subjects propel themselves forward with a more pronounced vertical dynamic of their gait pattern.

Using a strict Bonferroni correction, we found that speed, slumped posture, and lateral body sway remained gait characteristics that significantly differed in the depressed and never-depressed control group.

Results of the exploratory analysis indicate that there might be substantial differences between patients predominantly showing psychomotor agitation and patients showing psychomotor retardation. It would be a valuable task for future research to analyze in more detail the differences in gait characteristics of these subgroups.

A further point worth noting is the high degree of comorbid diagnoses (most of them anxiety disorders) in our sample of depressed patients. As epidemiological studies have repeatedly shown, MDD is a disorder characterized by high comorbidity $(25,26)$. We view the fact that the comorbidity rates in our sample are comparable to those reported in the literature as corroboration of the ecological validity of our sample. However, future research should also investigate noncomorbid depressed patients to assess gait patterns in more homogeneous samples.

A limitation of Study 1 is that depressed and never-depressed participants differ in two aspects that might influence gait parameters: depressed patients were heavier and were taking antidepressant medication. Medication might have influenced gait parameters by direct effects on neuronal systems that control gait. Moreover, it can also have an indirect effect on gait parameters by inducing weight gain in the depressed participants, a well-known side effect of antidepressant medication. We cannot rule out these possibilities because statistical control of potential covariates were not feasible (24). However, it should be noted that previous studies have found no effect of antidepressant medication on gait parameters (11) or small changes that were in the direction of the neverdepressed gait pattern reported in our study (i.e., increased gait speed, increased motor activity) $(12,27)$. However, one should be cautious in generalizing the current findings to unmedicated depressive individuals.

Our auxiliary analysis showed that although weight shows substantial covariation with some of the gait characteristics, the pattern of correlations is inconsistent with the notion that group differences are solely attributable to weight. Although body sway is correlated positively with weight and thus might contribute to group differences between depressed and neverdepressed participants, the direction of the associations of other variables with weight is inconsistent with this notion. In particular, the more pronounced vertical movements of heavier patients might instead have had the effect of decreasing group differences. Nevertheless, to make stronger conclusions in future research, it would be advisable to compare gait parameters of unmedicated depressed participants to a weightmatched comparison group.

Another line of research employing rigorous experimental methodology is to compare the gait patterns found in Study 1 with alterations in gait parameters associated with sad mood in 
a nonclinical sample. If gait parameters associated with major depression are comparable to those in sad mood, stronger conclusions about a convergent gait pattern associated with dysphoric mood states can be drawn. Correspondingly, in Study 2, we analyzed gait patterns post mood induction in a nonclinical sample. Using a within-subject design, we could also control for the effect of body weight and other parameters that may have covaried with the independent variable in Study 1.

\section{STUDY 2}

\section{METHODS}

\section{Participants}

Twenty-three undergraduate students enrolled in the Department of Psychology at the University of Bochum (21 females, 2 males) participated in the study. The mean age of the sample was 23.9 years (range $=$ 19-39 years). Students received credit for participation. The relative gender imbalance resulted from a much greater availability of female students and was unintended.

\section{Mood Induction and Procedure}

Based on Västfjäll's (28) review article on mood induction through music, two pieces of music were chosen to induce negative and positive mood. For the negative mood condition, we chose Barber's "Adagio for Strings" and for the positive affect condition, we selected Mozart's "Eine Kleine Nachtmusik: Allegro." Both pieces of music have been found to be effective in inducing mood in pre-, post-, and between-subject designs (29).

On arrival, participants were informed about the procedure and their written informed consent was obtained. They were also informed that they could terminate the experiment at any time. After preparation (i.e., after changing clothes and placing the markers), they were given the following instruction:

This experiment investigates gait patterns. At different time points during the experiment we will ask you about your mood. The experiment has two parts: First you will hear a piece of music for three minutes while you are sitting in a chair. You should get involved in the music to the maximum extent possible and let the music affect your mood. After that we will ask you about your mood. Then we would like you to walk on the red rug. You can choose your walking speed freely. While you are walking the music will continue. Again, please let the music affect your mood. After you have walked for a while we will again ask you about your mood. Then there will be a short break. In part two of the experiment you will again hear a piece of music.

The remainder of the instruction is parallel to the one used for the first part of the experiment. The order of music presentation was randomized for each participant. For each piece of music, the participants were seated with wireless headphones on a comfortable chair in the darkened laboratory as they listened to the track for 3 minutes.

After that, the participants had to walk up and down the red panel in the middle of the laboratory for approximately 8 minutes as the music presentation continued and motion data were being captured. We decided to continue music presentation during the walking and recording period to stabilize the mood induction. There was a break of approximately 15 minutes between the two conditions.

Participants rated their mood on a 1 to 9 scale $(1=$ extremely bad; $9=$ extremely happy) six times during the experiment: on arrival, after preparation (i.e., after changing clothes and placing the markers), after listening to the first piece of music for 3 minutes, and after walking with wireless headphones for the first time, after listening to the second piece of music, and after walking with wireless headphones for the second time. Similar analogue scales have been widely used in research on mood induction $(30,31)$. Motion data acquisition was identical to Study 1 . The data of Study 2 were gathered between December 2004 and February 2005.

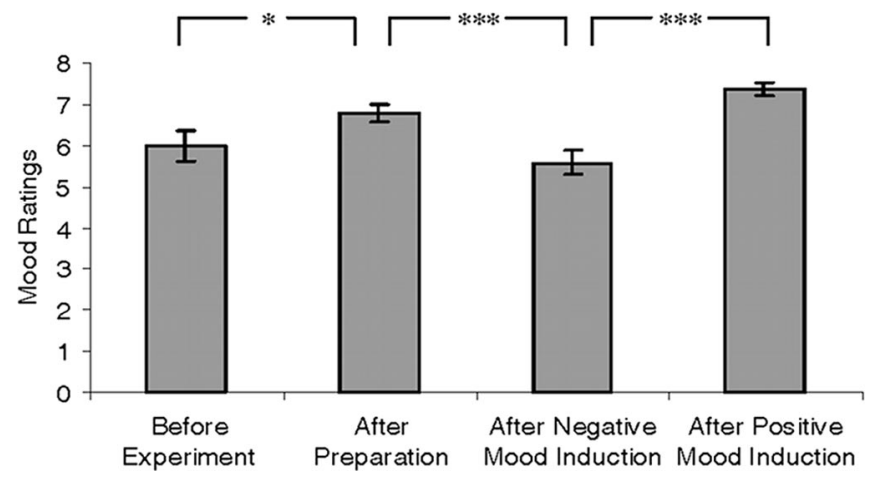

Figure 3. Study 2: Mood ratings for different times of assessment. Error bars represent one standard error. * $p<.05 ; * * * p<.001$.

\section{RESULTS}

\section{Mood Induction}

After participants arrived in the laboratory, they reported a mean affective state of $6.0(\mathrm{SD}=1.45)$; after completing the preparation for the experiment, affect ratings increased to 6.78 $(\mathrm{SD}=1.04)$. After negative mood induction, while they were seated on a chair for 3 minutes, the participants showed a mean mood rating of $5.57(\mathrm{SD}=1.38)$. After 8 minutes walking as sad music was continuously played, the participants showed a mean mood rating of $5.74(\mathrm{SD}=0.92)$.

Post happy mood induction for 3 minutes, the participants reported a mean affective state of $7.35(\mathrm{SD}=0.78)$. After walking as happy music was played, the participants showed a mean affective state of $7.26(\mathrm{SD}=1.39)$.

In a repeated-measures analysis of variance, the factor time of assessment revealed a highly significant effect regarding the rating of current affective state $(F(5,132)=10.02, p<$ $.001)$. Planned pairwise comparisons with mood levels after preparation (Figure 3) revealed significant differences for affective states before the experiment and after preparation $(p<.05)$. Ratings after preparation differed significantly from ratings after listening to sad music $(p<.001)$ whereas after preparation ratings only differed marginally from ratings after listening to happy music $(p=.10)$. Looking at differences with mood levels at baseline, we noted that a significant difference emerged between mood ratings before the experiment and after listening to happy music $(p<.001)$, whereas the differences between mood ratings before the experiment and after listening to sad music were not significant.

No significant differences were found for affect ratings in either affect condition after listening and after walking. Comparing ratings after listening to sad music with ratings after listening to happy music revealed a highly significant difference $(p<.001)$.

\section{Gait Patterns}

A linear discriminant function was constructed by regressing the independent variable (induction condition) on the parameters of the individual gaits as specified by the morphable model (23). An animation visualizing the linear discriminant function can be viewed at http://biomotionlab.ca/Demos/BMLdepression.html. 

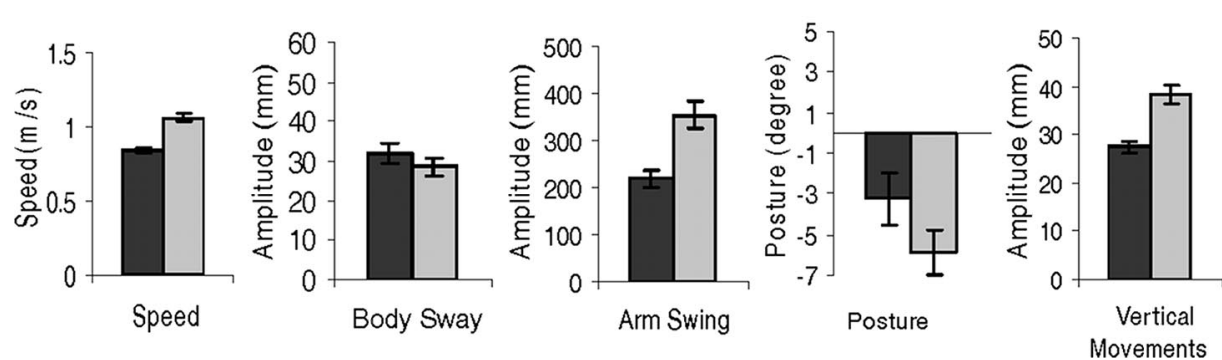

Figure 4. Study 2: Gait parameters associated with sad and happy mood. Error bars represent 1 standard error.

Visual inspection of the discriminant function again revealed that speed, arm swing, lateral body sway, posture of the upper body, and the amplitude of vertical movements of the upper body (measured in terms of vertical head motion) were discriminating features (see Figure 4 for means and standard errors of the variables). For Study 2, we first performed a repeated-measures analysis of variance (MANOVA) to assess differences between sad and positive mood simultaneously for all five gait characteristics. The MANOVA indicates highly significant differences between the sad and happy gait pattern $(F(5,18)=16.33, p<.0001)$.

Subsequent dependent sample $t$ tests revealed that all five features differed significantly in the sad and happy mood condition: participants in a sad mood walked more slowly (sad mood: $\mathrm{M}=0.84, \mathrm{SD}=0.13$; happy mood: $\mathrm{M}=1.06$; $\mathrm{SD}=$ $0.17, t(22)=8.79, p<.0001)$, showed reduced arm swing (sad mood: $\mathrm{M}=218.77, \mathrm{SD}=92.43$; happy mood: $\mathrm{M}=$ 352.86, $\mathrm{SD}=139.36, t(22)=7.97, p<.0001)$, showed greater lateral body sway ( $\operatorname{sad}$ mood: $\mathrm{M}=31.87, \mathrm{SD}=12.04$; happy mood: $\mathrm{M}=28.51, \mathrm{SD}=10.55, t(22)=-2.69, p<$ .05 ), showed a more slumped posture (sad mood: $\mathrm{M}=-3.25$, $\mathrm{SD}=6.08$; happy mood: $\mathrm{M}=-5.89, \mathrm{SD}=5.24, t(22)=-3.37$, $p<.01$ ) and reduced up-and-down movement of the head (sad mood: $\mathrm{M}=27.51, \mathrm{~S} D=5.16$; happy mood: $\mathrm{M}=38.45$, $\mathrm{SD}=8.93, t(22)=8.62, p<.0001)$. Following Bonferroni adjustment $(p<.01=.05 / 5)$, significance was maintained for speed, arm swing, posture, and vertical movements of the head.

As shown in the lower part of Figure 2, effect sizes (d) for differences between gait patterns associated with sad versus happy mood were large $(\mathrm{d}>0.80)$ for speed, arm swing, and vertical movements of the head.

\section{DISCUSSION}

Mood induction had pronounced effects on mood and gait patterns. Mood ratings revealed that the induction procedure was suitable and successful in producing pronounced differences between the sad and happy condition. However, differences between baseline/after preparation levels and mood after music induction revealed a slightly mixed picture (i.e., no significant difference between mood levels at baseline and mood levels after sad mood induction; only a marginally significant difference between after preparation mood ratings and mood levels after happy mood induction). Thus, one might speculate whether other mood induction procedures might have produced more pronounced effects with respect to baseline/after preparation mood levels.

The sad and happy mood conditions showed marked differences in gait parameters. Participants in the sad mood condition showed reduced speed, arm swing, vertical movements of the head and lateral swaying movements as well as a slumped posture. Using the stringent Bonferroni criterion, we found that lateral swaying movements no longer differed significantly between the two conditions, whereas differences in all other parameters remained significant.

However, although mood induction was successful and differences in gait patterns might be attributable to the mood induced by the music, an alternative explanation for the results of Study 2 might be that the rhythm and tempo of the music at least partially influenced walking speed and other gait characteristics. Although we cannot rule out this possibility, it should be noted that the study by Montepare et al. (2) investigating gait patterns associated with current sad and happy states yielded findings quite in keeping with those reported here. Results indicated that participants imagining sad scenarios showed reduced arm swing and a marginally reduced stride length. Posture ratings did not show significant differences between the sad and happy condition. However, because of the methodological limitations of the study by Montepare et al. (2) (e.g., dichotomous scales, untrained raters, head was not displayed in the video tapes), the latter result in particular should be interpreted with caution.

\section{GENERAL DISCUSSION}

The aim of our present research was to investigate one aspect of the embodiment of sadness and depression. In line with our notion that a convergent gait pattern characterizes sadness in nonclinical and major depression in clinical populations, we found converging gait characteristics in Study 1 and Study 2. Reduced walking speed, arm swing, vertical movement of the head, stronger lateral body sway, and a slumped posture characterize depressed and sad gait patterns. Using strict criteria, we found that speed and posture in particular unambiguously characterize the gait patterns of sad and depressed individuals. 
However, the interrelationship between the different gait characteristics that we identified in our study is not yet apparent. In the simplest case, parameters like body sway or slumped posture might all be caused by reduced walking speed. However, research on speed dependent changes in gait parameters in nonclinical participants have shown that, although the amplitudes of vertical movements of the trunk increase when velocity increases (parallel to our findings), lateral swaying movements of the upper body increase and participants show a more pronounced forward inclination of the trunk when walking speed increases (32). The latter two findings are in contrast to our results, where depressed and sad participants walked more slowly and showed an increase in lateral body sway and were more bent over when walking. Moreover, previous research has shown that posture variations by themselves can have noticeable affective and motivational consequences (33). These findings make it rather unlikely that all changes in gait characteristics that we found in our study could be attributable to variations in walking speed. Another more likely explanation for the covariation of parameters might be that the dysphoric emotional state causes reduced speed and, independently, other characteristics. To make stronger conclusions, however, future research should control speed experimentally (e.g., by using a treadmill) to decide whether different gait parameters vary independently of speed.

A limitation of our study is that the results do not permit unambiguous conclusions about the specificity of the gait patterns we obtained. Are the gait patterns we observed in Study 1 and Study 2 specific to depression and sad mood or are they, in addition, associated with a broader spectrum of negative affect like anxiety or anger? Results of Omlor and Giese (16) and Montepare et al. (2) indicated that angry walking - in contrast to the results we obtained for sadness and depression - is characterized by an increase of many joint angle amplitudes. Moreover, because of the biological function of anxiety (i.e., preparing the organism for action), it can be expected that anxiety should be associated with increases in motor activity. Nevertheless, although some evidence indicates that negative mood states like anger or anxiety might be associated with different gait patterns, further research is needed to draw stronger conclusions about the degree of specificity of the gait parameters we obtained in our present research.

In addition, discussions about the relationship between the two kinds of dysphoric mood states that were investigated in our research (i.e., sadness and clinical depression) have a long history (34). Whereas some researchers see only quantitative differences (in severity, duration, and clinical distress) between sadness and clinical depression, others stress qualitative differences. It seems safe to say, though, that a similar gait pattern characterizes both types of dysphoric mood states. However, the question whether there are also some (subtle) distinctions between the gaits of sad and depressed individuals needs further investigation.

A further limitation of our study is that the artificial environment of our laboratory might have affected the gait patterns of our participants. Because comparison groups were used in both studies, this is not a significant issue for interpreting our study. However, it might be worth considering whether future research should use complementary methods of investigating gait in more natural environments (e.g., videobased analysis of gait in ecological settings).

Despite these limitations, the convergent gait pattern we found in our two studies provides strong support for the notion that sadness and depression are embodied in the way people walk. The experimental design of Study 2 allows us to infer that mood states can have causal effects on the way people walk. The question whether the relationship between gait and mood is more complex (e.g., whether the way individuals walk can influence their mood) needs further investigation. Previous research (4) on the effects of simple movements and posture lend strong support to the assumption that bodily feedback loops affect emotions.

\section{REFERENCES}

1. Kraepelin E. Einführung in die Psychiatrische Klinik. Zweiunddreissig Vorlesungen [Introduction in the psychiatric clinic. Thirty-two lectures]. Leipzig: Barth Verlag; 1905.

2. Montepare JM, Goldstein SB, Clausen A. The identification of emotions from gait information. J Nonverbal Behav 1987;11:33-42.

3. Niedenthal PM. Embodying dmotion. Science 2007;316:1002-5.

4. Niedenthal PM, Barsalou LW, Winkielman P, Krauth-Gruber S, Ric R. Embodiment in attitudes, social perception, and emotion. Pers Soc Psychol Rev 2005;9:184-211.

5. Barsalou LW. Perceptual symbol systems. Behav Brain Sci 1999;22: 577-660.

6. Varela FJ, Thompson E, Rosch E. The Embodied Mind: Cognitive Science and Human Experience. Cambridge: MIT Press; 1991.

7. Pfeifer R, Bongard JC. How the Body Shapes the Way We Think. A New View of Intelligence. Cambridge: MIT Press; 2006.

8. Lakoff G, Johnson M. Philosophy in the Flesh: The Embodied Mind and Its Challenge to Western Thought. New York: Basic Books; 1999.

9. Teasdale JD. Multi-level theories of cognition-emotion relations. In: Dalgleish T, Power M, editors. Handbook of Cognition and Emotion. Chichester: Wiley; 1999.

10. Ekman P. Universals and cultural differences in facial expressions of emotion. In: Cole JK, editor. Nebraska Symposium on Motivation. Vol 19. Lincoln, NE: University of Nebraska Press; 1972.

11. Lemke MR, Wendorff T, Mieth B, Buhl K, Linnemann M. Spatiotemporal gait patterns during over ground locomotion in major depression compared with never depressed controls. J Psychiatr Res 2000;34: 277-83.

12. Paleacu D, Shutzman A, Giladi N, Herman T, Simon ES, Hausdorff JM. Effects of pharmacological therapy on gait and cognitive function in depressed patients. Clin Neuropharmacol 2007;30:63-71.

13. Sloman L, Berridge M, Homatidis S, Hunter D, Duck T. Gait patterns of depressed patients and normal subjects. Am J Psychiatry 1982;13:94-7.

14. Sloman L, Pierrynowski M, Berridge M, Tupling S, Flowers J. Mood, depressive illness and gait patterns. Can J Psychiatry 1987;32:190-3.

15. Wendorff T, Linnemann M, Lemke MR. Lokomotion und Depression [Locomotion and depression]. Fortschr Neurol Psychiatr 2002;70: 289-96.

16. Omlor L, Giese MA. Extraction of spatio-temporal primitives of emotional body expressions. Neurocomputing 2007;70:3034-45.

17. Wittchen H-U, Wunderlich U, Gruschwitz S, Zaudig M. SKID I. Strukturiertes klinisches Interview für DSM-IV. Göttingen: Hogrefe; 1997.

18. Association AP. Diagnostic and Statistical Manual of Mental Disorders. Washington: American Psychiatric Association; 1994.

19. Beck AT, Steer RA. Beck Depression Inventory-Manual. San Antonio: The Psychological Corporation; 1987.

20. Hautzinger M, Bailer M, Worall H, Keller F. Beck-Depressions-Inventar. Bern: Hans Huber Verlag; 1995.

21. Rabkin JG, Klein DF. The clinical measurement of depressive disorders. In: Marsella A, Hirschfeld R, Katz M, editors. The Measurement of Depression. New York: Guilford Press; 1987.

22. Troje NF. Decomposing biological motion: a framework for analysis and synthesis of human gait patterns. J Vis 2002;2:371-87. 
23. Troje NF. Retrieving information from human movement patterns. In: Shipley TF, Zacks JM, editors. Understanding Events: How Humans See, Represent, and Act on Events. New York: Oxford University Press; 2008.

24. Miller GA, Chapman JP. Misunderstanding analysis of covariance. J Abnorm Psychol 2001;110:40-8.

25. Kessler RC, Berglund P, Demler O, Jin R, Koretz D, Merikangas KR, Rush AJ, Walters EE, Wang PS. The epidemiology of major depressive disorder. Results from the national comorbidity survey replication (NCS-R). JAMA 2003;289:3095-105.

26. Kessler RC, Nelson CB, McGonagle KA, Liu J, Swartz M, Blazer DG Comorbidity of DSM-III-R major depressive disorder in the general population. Br J Psychiatry 1996;168:17-30.

27. Volkers AC, Tulen JH, Van Den Broek WW, Bruijn JA, Passchier J, Pepplinkhuizen L. 24-hour motor activity after treatment with imipramine or fluvoxamine in major depressive disorder. Eur Neuropsychopharmacol 2002;12:273-8.

28. Västfjäll D. A review of the musical mood induction procedure. Music Sci 2002;Special Issue:173-212.
29. Eich JE, Metcalfe J. Mood-dependent memory for internal versus external events. J Exp Psychol Learn Mem Cogn 1989;15:433-55.

30. Teasdale JD, Taylor R. Induced mood and accessibility of memories: an effect of mood state or of induction procedure? Br J Clin Psychol $1981 ; 20: 39-48$

31. Watkins E, Teasdale JD, Williams RM. Contextual questions prevent mood primes from maintaining experimentally induced dysphoria. Cogn Emot 2003;17:455-75.

32. Thorstensson A, Nilsson J, Carlson H, Zomlefer MR. Trunk movements in human locomotion. Acta Physiol Scand 1984;121:9-22.

33. Riskind JH. They stoop to conquer: Guiding and self-regulatory functions of physical posture after success and failure. J Pers Soc Psychol 1984; 47:479-93.

34. Blashfield RK. The Classification of Psychopathology: Neoknaepelinian and Quantitative Approaches. New York: Plenum Press; 1984.

35. James W. What is emotion? Mind 1984;19:188-205.

36. Cannon WB. The James-Lange theory of emotion: a critical examination and an alternative theory. Am J Psychol 1927;39:10-124. 